\title{
A MANAGEMENT SYSTEM AGAINST MAJOR RISK ACCIDENTS IN LARGE CONSTRUCTION SITES
}

\author{
Alessandro Carbonari, Angelo Biscotti, Berardo Naticchia, Federico Robuffo, Mario De \\ Grassi \\ Università Politecnica delle Marche, DACS Department - Division of Building Construction, \\ Ancona, Italy \\ alessandro.carbonari@univpm.it, a.biscotti@univpm.it, b.naticchia@univpm.it, \\ f.robuffo@univpm.it, m.degrassi@univpm.it
}

\begin{abstract}
The rates of job injuries and fatalities in construction sites are still too high, with respect to other industrial working places. Statistics show that there are a few main types of risk accidents causing the higher number of fatalities. This paper suggests the development of a novel low intrusive real-time monitoring system in support of health and safety management in construction sites and addresses its basic application cases. It is mainly intended as a support system for those inspectors in charge of the correct application of the measures dictated by the Health and Safety Plan. Its application in construction sites is rendered convenient by its low intrusiveness and easy expandability with the real work progress. Finally, first successful field tests carried out in a large construction site, regarding several residential block of flats under construction in an Italian town, are presented.
\end{abstract}

KEYWORDS: health and safety, real time monitoring, construction, mobile computing.

\section{INTRODUCTION}

Despite the decreasing trend of fatalities in construction sites recorded in the European area, however the construction industry still owns the poorest Health and Safety (H\&S) record of any major industry, being the probability of construction workers being killed higher than the average for all industries in Europe (EU Commission, 2008). Such statistics show that the major types of accidents are given by fatal falls to a lower level, falls of objects and collisions with transport means and mobile plants. Traditional practices like rewards, training or feedback communication have been shown to be only partially effective in construction sites, as they are non structured working places. Indeed, they are rendered more complex by the presence of different technologies, requiring the use of several types of equipments and resources at the same time. For that reason, accident occurrences can be only hardly predicted and each site has its own peculiarities. As a consequence, much research have been devoted to the development of intelligent control systems, adopting advanced communication technologies as the means for establishing new automated control systems. They are expected to have the capability of performing real time support to health and safety tasks in several ways: alerting in advance when fatalities are about to occur, automating dangerous operations and decreasing human interventions, improving performance management activities (Bowden et al., 2006). 
This paper is dealing moreover with the support that ICT based systems can give to inspectors in charge of safety. Current European legislation, and similarly also in many other countries, imposes that health and safety measures must be planned at the design level. The drawing of the H\&S plan is actually complete and adequate to discern all the safety measures that need to be adopted in order to avoid accidents, according to each site's expected work schedule. However, the real work schedule in the execution phase, is generally different from what panned, and presently made plan's updating releases, following from evidence collected during regular inspections, require a certain time lag to be implemented and usually are not in line with the fast evolving context in construction sites. This causes the H\&S plan to be not timely updated with the real work progress and with the new risks that could reasonably arise. As a consequence, all the work practices and policies reported in those plans and aimed at avoiding accidents among workers could be not applicable, because obsolete. This is particularly true for those risks raised by interferences among different squads working at the same time: if the site schedule changes, then the same happens to risk typologies, and the H\&S plan needs updating and conveying changes in safety policies to workers. Such a process could be manually manageable only in case several inspectors are continuously resident within the site. However this would be economically non convenient, hence the adoption of automated communication and localization technologies seems to be the only way. If their implementation will be capable of helping apply and update all the safety measures imposed by the H\&S plan, then all the workers will gain in awareness and incident rates are expected to dramatically decrease. Its feasibility has been shown by a number of contributions, which may be classified into two main types: studies aimed at conceiving safety targeted automated systems for risk prevention; experimental surveys, with the objective of verifying the applicability of monitoring systems in very complex and non structured working places, like constructions sites.

SightSafety is a very good example of conceptual model about a proactive system to prevent accidents deriving from plant and equipment operation, which suggests a combination of GPS technologies and MEMS sensors (Riaz et al., 2006). Other intelligent systems have been proposed, among them the mechatronics helmet to enhance worker awareness and communication capabilities in constructions sites (Abderrahim et al., 2005) and a mobile sensing device to detect workers approaching dangerous places with high risk of accidents (Ung-Kyun et al., 2009): this system would send alerts before the worker's entrance. Noteworthy efforts and step forwards towards reliable tracking technologies have been recently accomplished, too. Wireless Local Area Networks (WLAN), Ultra-Wide Band (UWB) and Indoor GPS positioning systems have been shown to perform reliably, but with different accuracy levels (Khoury et al., 2009); in particular, UWB (Teizer et al., 2008) and indoor GPS were found to be able to overcome the problems of multipath effects and obstructions. However, those installations were not immune to invasiveness, as they require the use of invasive cabling and time consuming calibration procedures. Also the recent tests on wireless and untethered UWB by (Cho et al., 2010) brought to conclude that those technologies require a long calibration set up. As a consequence, according to the present state of the art, they cannot be used in a diffuse manner all over the site; instead they could be valuably adopted for specific high level automated control tasks. In fact, their adoption all over the site would need several reconfigurations in time, which means also time consuming re-calibration, that would render their adoption non convenient.

It can be inferred that a basic system, capable of controlling in real time the correct application of the safety measures dictated by the H\&s plan, must be first of all non invasive 
and easily expandable and reconfigurable, without need for frequent re-calibration. Such kinds of system are generally also less accurate and less performing, however in this paper we will demonstrate that they allow the implementation of some basic automated safety policies, which can constitute a first important step towards further improvement in safety management. This idea will be highlighted in the following paragraph, where a description of the whole system will be provided. Then the hardware and software parts of the system will be described and tests results will follow. Conclusions and references end this paper.

\section{A NON INVASIVE SUPPORT SYSTEM TO HEALTH AND SAFETY MANAGEMENT IN THE EXECUTION PHASE}

\section{The role of the system for supporting health and safety management}

Actual H\&S plans have the main objective of establishing a set of instructions to employees and employers, regarding which work practices and safety policies must be applied in every phase of the work progress, in order to avoid hazards. These outputs are the consequence of previous risk identification and evaluation, which lead towards the definition of prevention and control actions. Relevant importance is reserved to potential hazards that may arise from the work place layout, which is valid until no changes occur during the execution phase, with respect to the planned schedule. That is why a support monitoring system is expected to help $H \& S$ coordinators to timely update the plan. That system can also be used for verifying the correct application of the plan's safety policies.

As it will be explained in the following, low intrusive systems nowadays means also low accuracy systems, meaning that they can estimate the area where every asset is located, with an average error of about $2 \mathrm{~m}$, but cannot estimate the exact location. However, those performances seem to be enough to perform basic controls, regarding the correct application of H\&S plan's safety measures. Table 1 lists three basic safety policies that are always included in H\&S plans, and which can be also be verified through the use of a non accurate monitoring system. As far as concerns the first policy, every employer must provide adequate training to his employees and transfer the list of workers he plans to involve in the site with the corresponding list of competencies. Hence, the system is expected to cross-check the rough position of every worker and the project's schedule to infer the activity under execution, finally verifying whether each worker has been trained for its task. Similarly it works for PPE items verification, with the only additional need of collecting the list of PPE items actually worn by employees, through the use of transponders. Finally, interference management can be accomplished by verifying whether the execution phase correspond to the planned schedule included in the H\&S plan.

Table 1: Correspondence between H\&S plan's policies and system automated controls

\begin{tabular}{|c|c|c|}
\hline $\begin{array}{l}\text { H\&S plan's } \\
\text { measures }\end{array}$ & Brief description & Support system's automated controls \\
\hline $\begin{array}{l}\text { Employees' } \\
\text { training }\end{array}$ & $\begin{array}{l}\text { Previous to perform his job, every } \\
\text { employee must receive adequate } \\
\text { training on applicable safety } \\
\text { measures }\end{array}$ & $\begin{array}{l}\text { From the list, attributes and position of } \\
\text { present workers, the system infer their job } \\
\text { and checks whether their training is } \\
\text { appropriate. }\end{array}$ \\
\hline
\end{tabular}


PPE wearing PPE items to be worn depend on By equipping PPE items with transponders the activities carried on the system can acquire also the types of PPE every worker is wearing and verify whether it is compliant with the H\&S plan

\begin{tabular}{lll}
\hline $\begin{array}{l}\text { Scheduling and } \\
\text { interferences }\end{array}$ & $\begin{array}{l}\text { In order to avoid dangerous } \\
\text { interferences, the presence of } \\
\text { workers must be compliant with the } \\
\text { site's scheduling }\end{array}$ & $\begin{array}{l}\text { The H\&S plan dictates the schedule for } \\
\text { activity execution, hence the system can } \\
\text { check that no unauthorized activities are } \\
\text { going on }\end{array}$
\end{tabular}

In order to perform such controls, an hardware/software platform must be installed in construction sites. We conceive it as a comprehensive system made up of a low level and non intrusive monitoring system deployed throughout construction sites, communicating via RF with both assets and with the main Server Application, where high level control logics are implemented and an interface to end users is provided, like in the scheme of Figure 1-a. As sites to be monitored are generally very large, then the use of several (expandable) subnetworks should be planned.

Based on this general idea, Figure 1-b depicts the logic approach we propose to perform interference prevention by means of this system. The H\&S coordinator must previously input on the site's layout the areas where every activity of the site's schedule is expected to be performed. Then the system acquires the ID and rough position of each worker present on site, it analyzes the job qualification for each worker and infer the kind of activities it is working on. In case it finds out that one ongoing activity is not in line with the site's schedule, then the system will send an alert to the H\&S coordinator. This could happen every time a worker is found outside the area where it was expected to work. In this way, the H\&S coordinator will be advised on time about the need to verify the real progress. Hence, this approach would work not only as a way for interference prevention, but also to signal changes of the work progress with respect to the planned schedule, allowing the H\&S coordinator to update the H\&S plan, thus improving the site's safety management, which is the first step to avoid the major number of incidents. In fact, many accidents occur because workers are dealing with unexpected risks due to unforeseen interferences, meaning that they had not been previously trained to deal with such situations. For that reason, verifying that each squad is working in line with the schedule would give the warranty that it knows how to face standard dangers that could reasonably be found.

Even additional checking can be managed through the use of non invasive but low accurate monitoring systems, according to the specific features of construction sites. For example, a wireless network interfaced with several sensors, would allow acquiring in real time many health indicators (e.g. sound level, dust concentration in air) and advise H\&S coordinators in case they overcome admissible thresholds. Similarly holds for cave-ins prevention, authorization to equipment usage, support to rescue operations in case of disasters etc.. However, there are some safety policies, like collision avoidance between machines in operation, being struck by means of transport or suspended loads and so on, where the adoption of accurate tracking systems is absolutely necessary. 


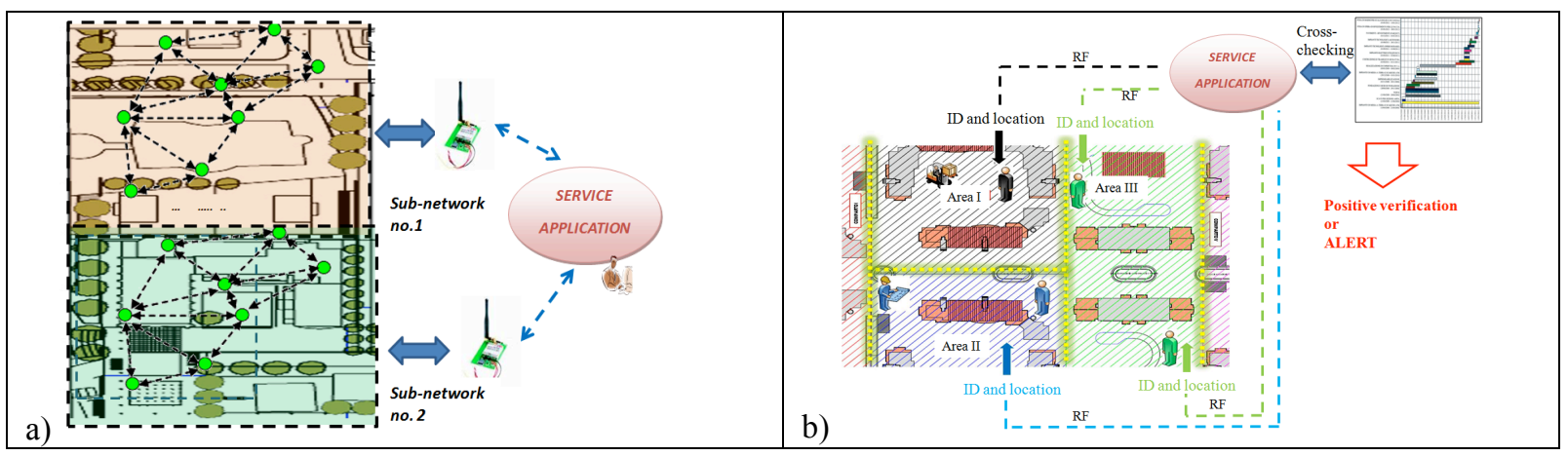

Figure 1: overall system architecture (a) and logic approach to automated interference prevention (b).

\section{TECHNICAL FEATURES OF THE SUPPORT SYSTEM}

\section{Low level monitoring sub-system}

The main feature needed from a system that must be deployed all over the whole area of construction sites is that it must not interfere with workers moving around in congested and dynamic situations. Hence, it must be made up of detached devices, with no cabling neither for communication nor for powering, hence battery powered, which means also very low power consumption to come out with at least a two year long power autonomy (considered as the average duration of work progress). In addition, their performances must be carefully analyzed and evaluated in such harsh environments, where RF signals could suffer many obstructions and wave interferences. Furthermore work progress imposes several reconfiguration of the site and requires frequent re-location of the network's devices in the area. To this aim, several types of low-power wireless communication networks are presently under development (e.g. Zigbee, Bluetooth, RFID etc...), capable of discerning the sub-area where workers and assets reside. They are also suitable to be interfaced with many sensor devices, besides acting as reliable communication systems.

For the purpose of our experimental campaign, a prototype of wireless communication sensor network, which is property of the Italian company Smart Space Solutions srl, was tested. It provides its features through hardware and software components based on the IEEE 802.15.4 standard medium access and Zigbee stack communication protocol. It owns the advantages of working in ISM band, being reliable, self healing and easy to deploy, having low cost and low power consumption. It is a very powerful system for communication purposes, and the whole network comprises three kinds of devices (Figure 2-a): one or more coordinators are used to initiate network formation, acting as 802.15.4 PAN coordinator (FFD); fixed devices with routing capabilities are installed at known locations of the area under monitoring, and are in charge of performing multi-hop routing of messages, following a pre-assigned balanced tree routing scheme, which can be modified at runtime; finally, end devices (or tags) are used as mobile nodes with associated IDs; they do not participate in routing but are worn or fixed on mobile assets to be tracked. Each fixed device joins a network in about $10 \mathrm{~ms}$ without any initial setup and the recovery and healing issues can be performed remotely, too. Localization is performed through the application of the "Weighted Centroid Localization" approach (Blumenthal et al., 2007), according to which the position of each tag (the same of the corresponding "asset") is computed as a weighted average value among the positions of the routers closest to the tag. Technically, a mobile node who wants to evaluate its position sends a broadcast message to all the routers in the network in its radio range. Each router 
sends to its PAN coordinator a short message whose payload contains its fixed coordinates and an indication of the message's RSSI received, measured in dbm. The RSSI indicator is then used by a central application server, directly connected to the PAN coordinator via TCP/IP protocol, to estimate the weighting coefficient associated to each router's coordinates for implementing the Weighted Centroid Localization algorithm.

Low power features of the network are assured thanks to a reduced routers' duty cycle in asynchronous mode. Each router remains in sleep mode for reducing power consumption until it has to route a message or reply to a request. In these cases an asynchronous signal wakes it up from sleep and allow it to work without wait for a sync. The localization hardware engine and the low power communication capabilities, combined with its selfhealing property, make this wireless sensor network extremely suitable in dynamic environments, in respect of other tracking technologies.

\section{The software interface}

The middle layer of the whole system was programmed so that the whole area of the site under testing was monitored as a set of sub-areas, defined trough the input of geographical coordinates. Then, a list of authorized workers was assigned to each sub-area, associated to the corresponding time windows they are authorized to operating herein. An iterative control was then executed at each data record, in order to verify whether any worker accessed a non authorized sub-area; in the positive case an alert would be sent. A friendly user interface was designed, based on Windows platform ${ }^{\mathrm{TM}}$, to provide the following features: real time monitoring of the assets resident on site; classification of the resources according to predefined categories (personnel and equipment) and to the inputted sub-areas; on screen selection of the visualized tags to list its related features (ID, position, type of resource, local coordinates and its history); alert management (Figure 2-b). To be noticed that the visualization map depicts both tags' estimated position and the corresponding sub-area. From the user's perspective this software could be used both to visualize in real time (and from remote location) all the resources present on site and to assist decision making following any sent alarm: as it is capable of highlighting the sub-area accessed by non authorized assets, then it helps deciding the best counter action to be taken. Sometimes, such situation could determine a re-organization of work schedule to avoid dangerous interferences; other times an updating of the H\&S plan could be enough, in case changes do not add further risks.

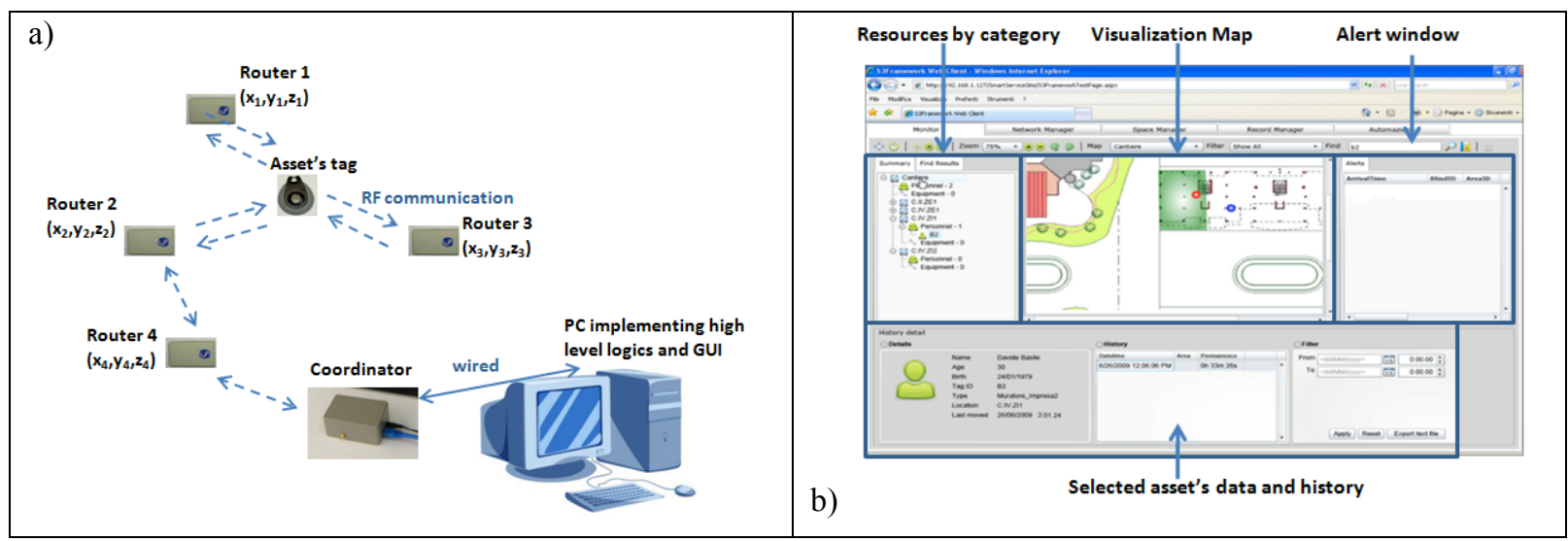

Figure 2: low-level monitoring system's architecture (a) and software interface (b). 


\section{EXPERIMENTAL CAMPAIGN}

\section{System setup}

The experimental campaign was conducted in a portion of a large construction site, concerning the erection of seven blocks of flats in an Italian town, where very often several squads work at the same time in each block of flats. As the whole commitment is divided into five subareas (Figure 3-a), then managing health and safety against unauthorized interferences is a key challenge. In the entire residential area will be erected two types of blocks: each type 1 block hosts 72 flats distributed from the ground floor to the third floor, where flat sizes vary between 40 an $136 \mathrm{~m}^{2}$; they are served by eight lift blocks. Each block of type 2 includes 48 flats, distributed from the ground floor to the third floor around three lift blocks, and with a size limited between 51 and $125 \mathrm{~m}^{2}$. Both types of buildings have a reinforced concrete made bearing structure, with concrete slab floors and masonry type walls. The network was installed in the red colour circled portion of the site marked in Figure 3-a and zoomed as in Figure 3-b, crossing sub-areas no. II, III and IV. This choice was done in order to realistically simulate the case of workers crossing from one sub-area to another and checking whether the system is able to sniff unauthorized accesses.

The routers of the network have been installed in the site as shown in Figure 3-c. For an entire view of their deployment, please refer to Figure 4-a. To be noticed that, thanks to the use of wireless and untethered devices, their installation is easy and non invasive. In fact, routers are packed inside water tight, relatively small $(0.08 \times 0.1 \times 0.05 \mathrm{~m}$ sized $)$ and light plastic boxes, which can be easily fastened through nails, glue or slender wires to walls, concrete surfaces, scaffold's columns and so on. Thus, also their relocation in case of network's expansion or reconfiguration would be seamlessly pursuable. Then, one coordinator and the PC running the Server Application have been located in a flat of the block under construction inside sub-area II, and three tags have been given to as many members of the crew performing the experimental campaign.

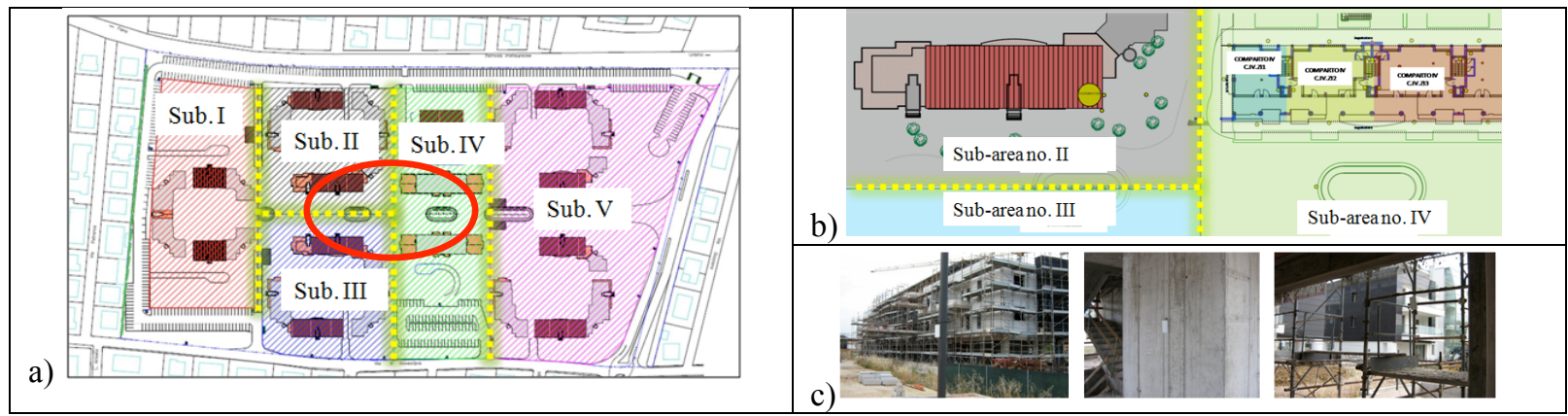

Figure 3: construction site hosting the experimental campaign (a), zoom on the testing area (b) and some photos of the routers installed (c).

\section{Testing and discussion on results}

The experimental campaign was carried out for two main purposes: showing that the monitoring network is able to reliably perform zoning and that the Application Server is capable of signalling in real time dangerous unauthorized accesses.

After having installed 15 routers (blue and grey coloured) and one coordinator in the locations depicted in Figure 4-a, then one member of the crew was asked to walk along the 
most hidden path of the monitored area, that is to say inside the building in sub-area IV. Figures 4-b and 4-c compares the real position of the worker and the one shown by the system, which was enough to conclude that it reliably recognizes the area of interest in the site. To be noticed that the frequency of the end device was equal to $5 \mathrm{~s}$, hence this is approximately the time lag associated with such recordings. In any case it is considered as acceptable, given the scope of the system, which is not aimed at continuously tracking assets' movements, but more oriented towards control of the site's regular working organization. The network's deployment did not suffer obstructions caused by reinforced concrete walls and by metallic scaffolds. An area about $(60 \times 30) \mathrm{m}$ large, including both outdoor and indoor environments was covered with the use of 15 routers; it follows that on average one router every $120 \mathrm{~m}^{2}$ is enough at this site progress, when only the reinforced concrete frame is finished and wall erection is about to start. In addition, some redundant sensors have been positioned, because it is considered to be a good practiced against networks faults. Obviously, network expansion would be an easy task, as explained above.

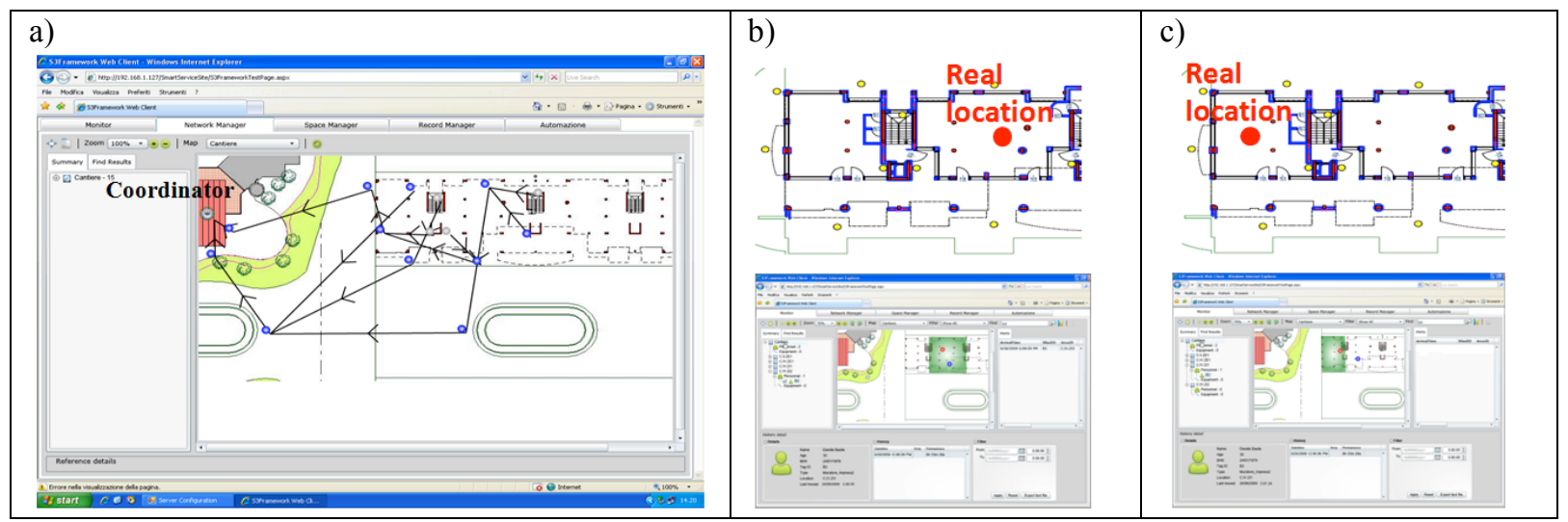

Figure 4: Routers positioning (a), comparison between real and estimated positions in the first trial $(\mathrm{b}, \mathrm{c})$

The second trial had the objective of validating the software capability to timely signal any non authorized access, that in this case was planned to take place from sub-area IV into subarea II. A guy was equipped with one tag hung from his belt, and then dressed like a worker and asked to move from the initial point to the destination depicted in Figure 5-a. Another operator filmed in real time the screenshots of the software interface, and two of them are reported in Figure 5-b, as they demonstrate the two main moments when the operator is walking within sub-area IV and then within sub-area II. In the second screenshot the alert sent by the software after its entrance is red circled and witnessed by the second operator; it appeared between five and six seconds after the first operator crossed the border line. Another proof of the trial is given by the shot sequence shown in Figure 5-c. Also in this case the system did not suffer the presence of several squads at work and of equipment in operation, but it reliably tracked ongoing movements.

Also the software interface resulted to be a very powerful tool for site management: it worked well to check which resources are present on site at every time moment, and also to send alerts to the H\&S coordinator when any employee is not behaving as expected. The system, even if with some limitations in terms of tracking accuracy, was shown to assist properly when dealing with Health and Safety. 


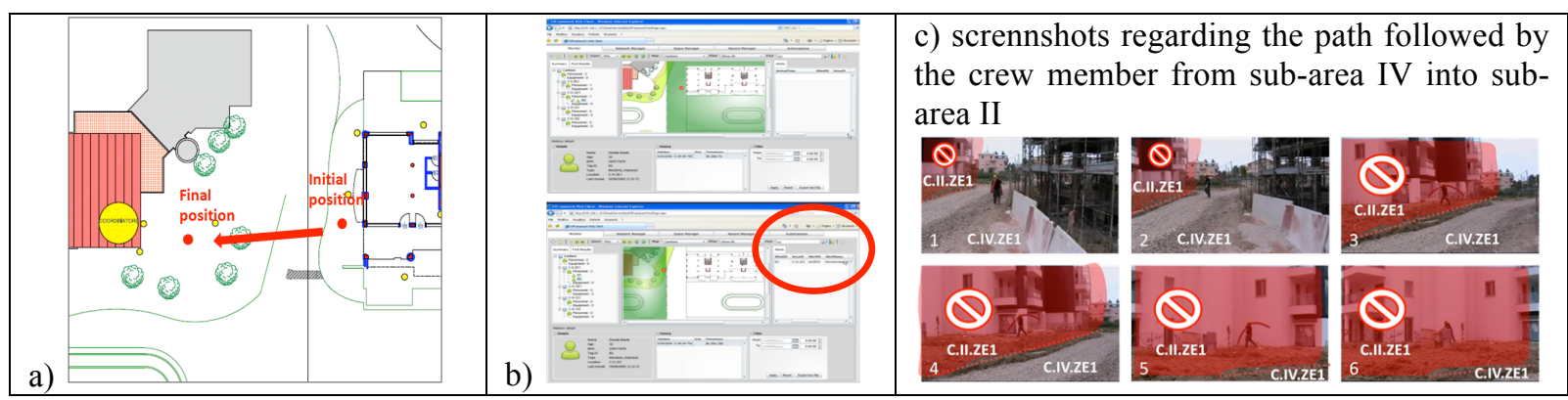

Figure 5: path followed by a crew member from sub-area IV into sub-area II (a), corresponding visualization in the interface (b) and screenshots (c)

\section{CONCLUSIONS}

The hardware/software prototype developed and described in this paper, was shown to be a powerful instrument for supporting health and safety management in construction sites. It would act as an assistant man for the H\&S coordinator, which cannot be always present on site. Hence, an intelligent tools for monitoring possible changes with respect to initial schedule must be adopted, in order to avoid that unexpected interferences would turn into fatalities. The main positive features shown by the system were its low invasiveness, due to the complete absence of cabling and to its long term self-powering when battery powered. In addition, also the small size of its devices makes its initial deployment and next reconfiguration needs a rather easy task to be pursued.

Based on this monitoring system, a first type of high level logic has been developed, in order to provide a practical example of the support it would give to H\&S coordinators. Through its adoption, it would be possible to continuously check whether H\&S plan's measures are correctly applied, and intervene soon in the opposite case. This paper suggests also other basic policies, always dictated in H\&S plans, that can be controlled through the use of a similar set up, and that would dramatically decrease incident trends.

Further logics and more testing in real construction sites will be performed, in order to evaluate the long term performances and reliability of the system, with particular concern to its resistance to harsh and dynamic conditions typical of these working places.

\section{REFERENCES}

EU Commission - Directorate-General for Employment, Social Affairs and Equal Opportunities, (2008) Causes and circumstances of accidents at work in the EU, Luxembourg: Office for Official Publications of the European Communities, ISBN 978-9279-11806-7.

Sarah Bowden, Alex Dorr, Tony Thorpe, Chimay Anumba (2006) Mobile ICT support for construction process improvement", Automation in Construction, vol. 15, 664-676.

Z. Riaz, D.J. Edwards, A. Thorpe (2006) SightSafety: A hybrid information and communication technology system for reducing vehicle/pedestrian collisions, Automation in Construction, vol. 15, $719-728$. 
M. Abderrahim, E. Garcia, R. Diez, C. Balaguer, (2005) A mechatronics security system for the construction site", Automation in Construction, vol. 14, 460-466.

Ung-Kyun Lee, Joo-Heon Kim, Hunhee Cho, Kyung-In Kang, (2009) Development of a mobile safety monitoring system for construction sites, Automation in Construction, Vol. $18(3), 258-264$.

Hiam M. Khoury 1, Vineet R. Kamat, (2009) Evaluation of position tracking technologies for user localization in indoor construction environments, Automation in Construction, vol. 18, 444-457.

Teizer, J.; Lao, D.; Sofer, M. (2008) Rapid automated monitoring of construction site activities using Ultra-WideBand, Proceedings of the 24th International Symposium on Automation and Robotics in Construction - ISARC 2007, September 19-21, Kochi, India.

Yong K. Cho, Jong Hoon Youn, Diego Martinez (2010) Error modeling for an untethered ultra-wideband system for construction indoor asset tracking, Automation in Construction, vol. 19(1), 43-54.

Jan Blumenthal, Ralf Grossmann, Frank Golatowski, Dirk Timmermann. (2007) Weighted Centroid Localization in Zigbee-based Sensor Networks. Proceedings of the IEEE International Symposium on Intelligent Signal Processing (WISP 2007), Madrid, Spain. ISBN: 1-4244-0830-X. 